ISSN 2447-9071

doi https://doi.org/10.36414/rbmc. v5i13.23
Contato para correspondência: Lucas Fernando Souza Pereira

E-mail:

lucasfernadospereira@gmail.com

Conflito de interesse: Não

Financiamento: Conselho Nacional de Desenvolvimento Científico e Tecnológico (CNPq)

Recebido: 11/11/2019

Aprovado: 12/12/2019

\section{Avaliação da prevalência dos casos de sífilis na população da vila mutirão atendida pelo laboratório clínico PUC Goiás}

\author{
Evaluation of the prevalence of syphilis cases in the population of \\ the mutirão village attended by the PUC Goiás clinical laboratory
}

\author{
Sérgio Henrique Nascente Costa ${ }^{1,2,3}$ \\ ${ }^{1}$ Pontifícia Universidade Católica de Goiás - PUC Goiás \\ ${ }^{2}$ Faculdade da Polícia Militar - FPM \\ ${ }^{3}$ Universidade Federal de Goiás - UFG
}

Lucas Fernando Souza Pereira', Matheus de Oliveira Cardoso' ${ }^{1}$, Frank Sousa Castro ${ }^{1,2}$

\section{Resumo}

O presente estudo buscou identificar a prevalência dos casos de sífilis, delineando o perfil epidemiológico associado na Vila Mutirão. Trata-se de um estudo retrospectivo baseado na análise dos dados do Posto de Coleta da UABSFVila Mutirão de Goiânia/GO. Foram verificados os resultados dos exames complementares solicitados para diagnóstico da sífilis (VDRL e FTA-abs) e o perfil epidemiológico dos pacientes. Os resultados coletados e analisados através do software Epi Info, comnivelde significância inferiora $5 \%(p<0,05)$. Foram analisados, referentes aos anos de 2017 e2018, um total de 6.386 exames. No ano de $2017,36,7 \%$ era do sexo masculino e $63,3 \%$ do sexo feminino e, em 2018, 58,8\% do sexo masculino e $41,2 \%$ do sexo feminino. Com relação ao VDRL, em 2017, 87,7\% detinham resultado não reagentee 12,2\% tiveram comoresultado exames reagentes. Em 2018obteve-se $81,4 \%$ nãoreagentes e 18,5\% reagentes. Dos pacientes reagentes, $48,6 \%$ também realizaram a pesquisa do FTA-abs IgMe IgG. Quanto ao FTA-abslgM, 46,3\% tinham amostra reagente para a presença deste anticorpo, 34,2\% tinham amostra não reagente e 19,4\% indeterminado. No que tange ao FTA-abs lgG, 91,7\% tiveram resultado reagentee $8,3 \%$ obtiveram não reagente. Com relação aos pacientes reagentes ao VDRL em 2018, 16\% também realizaram a pesquisa doFTA-abs IgMe 25,2\% a pesquisa doFTA-abs IgG. Quanto ao FTA-abs IgM, 31,6\% tinham amostra reagente, 8\% não reagente para tal e 60,3\% indeterminado. Quanto ao FTA-abs lgG, 98,6\% tiveram resultado reagentee 1,4\% obtiveram a resposta não reagente paraeste anticorpo. Épossível concluirque a populaçãoestudada seencontra sujeita a um crescimento de infecção por síflis, principalmente a população do sexo masculino adulto-jovem e ressalta-se a necessidade de estudos com delineamentos populacionais mais rígidos.

Palavras-Chave: Prevalência, Síflis, VDRL, FTA-abs.

\begin{abstract}
The present study aimed to identify the prevalence of syphiliscases, outlining the associated epidemiological profile in Vila Mutirão. This is a retrospective study based on analysis of data from the UABSF Collection Station Vila Mutirão de Goiânia/GO. The results of the complementary exams requested for the diagnosis of syphilis (VDRL and FTA-abs) and the epidemiological profile of the patients were verified. The results were collected and analyzed using Epi Info software, with a significancelevel below $5 \%$ ( $p<0.05$ ). A total of 6.386 exams were analyzed for 2017 and 2018. In 2017, 36.7\% were male and 63.3\% female, and in $201858.8 \%$ male and $41.2 \%$ female. Regarding the VDRL, in 2017,87.7\% hadnonreactive results and $12.2 \%$ had reactive tests. In $2018,81.4 \%$ non-reactants and $18.5 \%$ reagents were obtained.Of the reactive patients, $48.6 \%$ also performed the FTA-abs IgM and IgG research.Regarding FTA-abs IgM, 46.3\% had a reagent sample for the presence of this antibody, 34.2\% had a nonreactive sample and $19.4 \%$ undetermined.Regarding FTA-abs Ig G, 91.7\% had a reagent result and $8.3 \%$ had a non-Reagent response. Regarding patients reacting to VDRL in 2018, 16\% also performed the FTA-abs
\end{abstract}


IgMsurvey and $25.2 \%$ performed theFTA-abs IgGsurvey. Regarding FTA-abs IgM, 31.6\% had a reagent sample, $8 \%$ nonreactive and $60.3 \%$ undetermined. As for FTA-abs lgG, $98.6 \%$ had a reagent result and $1.4 \%$ had a nonreactive response for this antibody. It can be concluded that the study population is subject to a growth of syphilis infection, especially the young adult male population and the need for studies with more rigid population designs is emphasized.

Keywords: Prevalence, Syphilis, VDRL, FTA-abs.

\section{Introdução}

A sífilis é uma doença infecciosa crônica, de transmissão sexual ou vertical, que pode ficar por grandes períodos sem sintomas clínicos e atingir múltiplos sistemas, produzindo lesões cutâneas, mucosas, cardiovasculares e nervosas. O agente causador é a bactéria Treponema pallidum e sua transmissão ocorre por contato direto com lesões abertas ou mucosas intactas. O Treponema pallidum, por via linfática e/ou hematogênica, invade rapidamente todo o organismo, tornando a infecção generalizada em poucas horas, mesmo quando a sintomatologia ainda é local ${ }^{1,2}$.

O cancro sifilítico ou cancro duro é a primeira manifestação clínica da síflis adquirida. Após uma incubação de duas a quatro semanas, ocorre uma resposta inflamatória local, a qual caracteriza a lesão do cancro (única, exulcerada, de fundo limpo, cor de carne, cujas bordas descem para o fundo, associada a um exsudato seroso escasso, rico em treponemas, indolor, com consistência dura e cartilaginosa à palpação), que passará pela resolução espontânea em aproximadamente um mês'. Esta característica da resolução dificulta o diagnóstico precoce e a busca por tratamento, devido à crença errônea dos pacientes de que, pelo o desaparecimento da lesão, a infecção foi solucionada.

O período secundário da doença surge geralmente após seis semanas e pode durar até dois anos após a infecção, com manifestações generalizadas como as cutaneomucosas chamadas de roséola sifilítica, o condiloma plano, as placas mucosas e a alopecia em clareira. Após esse período, desaparecem completamente os sinais e sintomas clínicos, iniciando-se a chamada latência sifilítica, período no qual as provas sorológicas detectam a existência da síflis sem que nenhuma manifestação ocorra. Posteriormente, em um terço dos casos, surge o período terciário da enfermidade, caracterizado por manifestações cutâneas e/ou viscerais'.

Ademais, vale ressaltar a importância do cuidado com a ocorrência da síflis em mulheres, especialmente visto ser uma doença com graves consequências para a grávida e para o seu feto, pois, quando transmitida intraútero, leva à sífilis congênita ${ }^{4}$.A síflis na gravidez pode causar problemas de saúde nas mães e nas crianças, estimando-se que entre 50 a $80 \%$ das gestações afetadas (dependendo do estágio materno) resultam em afecção fetal ou infantil grave, desfavorável, como morte fetal, morte neonatal, prematuridade ou infecção congênita do neonato 5 .

Segundo a Organização Mundial de Saúde (OMS), a sífilis afeta um milhão de gestantes por ano em todo o mundo, fato este que se desdobra em mais de 300 mil mortes fetais e neonatais e coloca em risco de morte prematura mais de 200 mil crianças. Na América Latina, cerca de 166.000 a 344.000 crianças nascem com síflis congênita, número esse que chama atenção e evidencia falhas na atenção básica e pré-natal, já que, atualmente, a síflis é uma doença de fácil prevenção, diagnóstico e tratamento ${ }^{4}$.

Nos últimos anos, no Brasil, constatou-se um aumento contínuo dos registros de casos de sífilis adquirida ou congênita, fato sucedido em virtude do maior aporte concedido pelas políticas públicas de saúde ao rastreamento e combate da doença. Entretanto, apesar das medidas governamentais para que a doença pudesse ser detectada e tratada de forma mais eficiente, como a instauração da notificação compulsória da sífilis adquirida a partir de 2010, os indicadores demonstram que não vem ocorrendo a associação temporal da detecção da infecção e a respectiva cura dos casos identificados ou a devida prevenção da sífilis congênita durante a gestação 5 .

Aprimorando o sistema de vigilância, instituindo o aumento da cobertura de testagem e ampliando o uso de testes rápidos na atenção básica, o Departamento de Vigilância, Prevenção e Controle das Infecções Sexualmente Transmissíveis (IST), Aids e das Hepatites Virais (DIHAV) do Ministério da Saúde buscou enfrentar as mazelas advindas da realidade da sífilis no país, mas percebeu-se, ao contrário do que era esperado, que os casos identificados eclodiam mais a cada ano de forma constante, crescente e sem resoluções práticas ${ }^{5}$.

Segundo o Ministério da Saúde, pelo Boletim de Vigilância Epidemiológica de 2017, os casos de síflis em gestantes e os de sífilis congênita no Brasil triplicaram entre 2010 e 2016 e os casos de sífilis adquirida passaram de 2,0 casos por 100 mil habitantes para impressionantes 42,5 casos por 100 mil habitantes no mesmo período. Em 2016, foram notificados 87.593 casos de sífilis adquirida, 37.436 casos de síflis em gestantes, 20.474 casos de sífilis congênita e 185 óbitos no Brasil pela sífilis, fora 
os milhares de pacientes afetados por morbidades vinculadas à doença, números esses que mostram a imprescindibilidade de medidas que garantam uma atenção em saúde eficaz no que diz respeito ao combate à síflis 5 .

Deve-se ter em mente que a sífilis é considerada uma das principais doenças sexualmente transmissíveis coinfectantes, sendo, por exemplo, a mais prevalente em gestan-tes portadoras do HIV e podendo, além dos eventos associados à sua própria fisiopatologia, facilitar a transmissão vertical da AIDS 6 . Assim, para o ano de 2018, o DIHAV implantará o projeto de resposta rápida a sífilis nas redes de atenção para reduzir a sífilis adquirida e em gestantes, e eliminar a síflis congênita no Brasil através de uma resposta colaborativa que articule os pontos de atenção à saúde, os setores sociais e a comunidade para fortalecer a resolução desses agravos ${ }^{5}$.

Incrementando tal cenário, através do Boletim de Situação Epidemiológica da Sífilis, divulgado pela Secretaria de Saúde do Estado de Goiás em 2017, nota-se que a realidade estadual acompanha a do país: um crescimento intenso e constante dos casos de infecção. Entre 2013 e 2016, no estado, constatou-se um aumento de $453 \%$ de síflis adquirida, $31 \%$ de síflis em gestantes e $107 \%$ de síflis congênita, números que não somente impressionam os órgãos de vigilância, mas também se refletem em agravos substanciais e profundos referentes à saúde e à qualidade de vida da população em geral ${ }^{7}$.

Na tentativa de contornar a problemática, foram levantados alguns pontos de falhas do sistema de atenção do estado e constatou-se que vem ocorrendo: o acesso tardio ao pré-natal (somente $20 \%$ das gestantes com sífilis tiveram o diagnóstico realizado no primeiro trimestre); o diagnóstico extemporâneo (aproximadamente $40 \%$ das mães de crianças com sífilis congênita tiveram o diagnóstico no momento do parto ou após este); o tratamento inadequado $(93,8 \%$ das mães de crianças com síflis congênita tiveram o tratamento inadequado, considerando a administração de outro medicamento fora a penicilina benzatina, o tratamento no período inferior a 30 dias antes do parto e o não tratamento do parceiro sexual como inadequações) e o preenchimento errôneo da ficha de notificação ( $23 \%$ das gestantes com síflis não tiveram classificação clínica), fatos estes que dificultam o enfrentamento da situação atual ${ }^{7}$.

Portanto, apesar das medidas já instauradas no combate ao aumento da sífilis no estado, como a instituição do Comitê de Investigação de Transmissão Vertical de Síflis, HIV e Hepatites B e C, por meio da Portaria $n^{\circ} 512 / 2017$, nota-se que muito ainda precisa ser conquistado para que as falhas identificadas não reverberem e provoquem graves consequências para a saúde da população ${ }^{7}$.

Desta forma e perante tamanha adversidade inserida nesse fenômeno, o presente estudo realizou um levantamento dos ca- sos da região analisada e do perfil epidemiológico, com o intuito de auxiliar no enfrentamento do problema atual, considerando não apenas a demanda das realidades nacional e estadual, mas também a do próprio serviço de saúde do local de estudo.

\section{Métodos}

Trata-se de um estudo retrospectivo com análise dos dados do Posto de Coleta da UABSF Vila Mutirão da região noroeste de Goiânia/GO. Foram verificados os resultados dos exames complementares solicitados para diagnóstico da síflis (VDRL e FTA-abs) na rede de estratégia de saúde da região e o perfil epidemiológico dos pacientes. Foram selecionados todos os pacientes da região Noroeste de Goiânia/GO que, por indicação clínica das equipes de saúde da região, passaram pela triagem ou pelo diagnóstico para infecção por sífilis através de exames no Laboratório Clínico PUC Goiás (LAC-PUC Goiás). Analisou-se todos os resultados dos testes não-treponêmicos e treponêmicos realizados no laboratório supracitado e advindos da região a ser estudada. O diagnóstico da sífilis, após a suspeita clínica, é realizado através destes testes ${ }^{1,8}$.

Foram analisados os exames realizados entre Janeiro de 2017 e Dezembro de 2018. Quanto aos exames VDRL foram observados se estes eram não reagente ou reagente. Quando reagente, verificado a titulação da reação, sendo que foram considerados como sugestivos de infecção quando maiores ou iguais que 1/4. Quanto ao FTA-Abs observou-se apenas se estes eram reagentes ou não reagentes. A presente pesquisa foi analisada pelo Comitê de Ética em Pesquisa PUC Goiás (Parecer $n^{\circ} 235.376$ de 20/03/2013) e foi aprovada.

O exame mais utilizado de teste não treponêmico é o VDRL (Veneral Disease Research Laboratory) com uso recomendado apenas para triagem dos pacientes com suspeita de infecção, por ser sensível para deteç̧ão, mas, no entanto, não é específico, podendo gerar resultados falso-positivos. O VDRL é reativo a partir da segunda semana após o surgimento do cancro duro, reduzindo os títulos após o primeiro ano e negativando após uma média de 12 meses depois do tratamento'.

Por outro lado, o teste treponêmico é um exame qualitativo e específico para o treponema, sendo o FTA-Abs (Fluorescent Treponemal Antibody Absorption) o mais frequentemente usado, já que detecta anticorpos lgM elgG contra os antígenos das espiroquetas causadoras da sífilis, confirmando o contato já na primeira semana da infecção após o contágio. Este teste é então utilizado para confirmar a infecção dos pacientes que possuem um teste de VDRL reagente, comprovando que estes não estão vinculados a um falso-positivo, mas sim à infecção propriamente dita ${ }^{1,8}$.

A análise estatística foi realizada a partir de um banco de dados do sistema de gestão laboratorial PCLAB ONLINE ${ }^{\circledR}$, que 
possui as informações referentes ao Posto de Coleta da UABSF Vila Mutirão da Região Noroeste de Goiânia-GO. Todas as informações obtidas foram mantidas em sigilo de pesquisa e nenhuma informação será divulgada individualmente, sendo preservada a confidencialidade das informações. Os resultados coletados foram tabulados em uma planilha no Microsoft Excel 2010. As análises foram executadas por meio da utilização do software Epi InfoTM versão 3.5.3, com nível de significância inferior a $5 \%(p<0,05)$.

\section{Resultados}

Foram analisados, de forma geral, os exames referentes ao rastreamento da síflis de 1.815 pacientes realizados no ano de 2017 e de 4.571 pacientes do ano de 2018, alcançando um total de 6.386 exames analisados pela presente pesquisa. Dentre a população pesquisada, no ano de $2017,36,69 \%$ (666/1815) era do sexo masculino e $63,30 \%$ (1149/1815) do sexo feminino. Por outro lado no ano de 2018, a população foi composta por $58,82 \%$ (2689/4571) do sexo masculino e $41,17 \%$ (1882/4571) do sexo feminino (Figura 1).

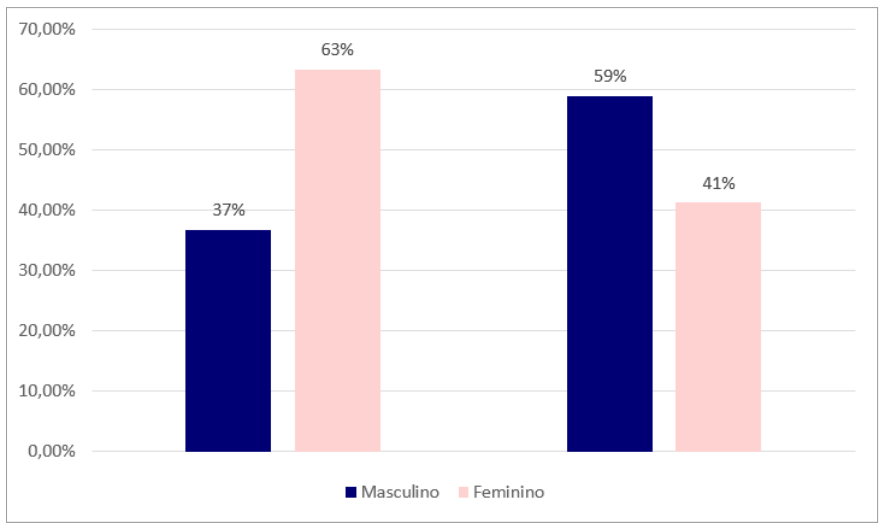

Figura 1: Comparativo percentual entre os sexos dos pacientes pesquisados em 2017 e 2018.

Ainda vinculado ao sexo dos pacientes pesquisados, obtevese diferença significativa quando os mesmos foram comparados entre os resultados reagente e não reagente à pesquisa do VDRL. Aplicou-se o teste chi-quadrado com o número total de pacientes reagentes e não reagentes e com os valores totais de pacientes do sexo masculino (764 reagentes e 2.591 não reagentes, totalizando 3.355 pacientes) e feminino (308 reagentes e 2.723 não reagentes, totalizando 3.031 pacientes), referentes tanto ao ano de 2017 quanto 2018. Tal relação evidenciou uma maior taxa de infecção em pacientes do sexo masculino durante o período estudado $(p<0,0001)$.
Com relação a idade dos pacientes pesquisados, dos vinculados a exames realizados em 2017, obteve-se uma média e desvio padrão de 37,9 916,3 anos, com predomínio de distribuição nos intervalos de 21 a 30 anos $(25,4 \%)$ e de 31 a 40 anos $(24,2 \%)$. No que diz respeito a idade dos pacientes pesquisados em 2018, chegou-se a uma média e desvio padrão idade de $34,8 \pm D P=$ 14,1 , com predomínio de atendimento presente nos intervalos de 21 a 30 anos (40,4\%) e de 31 a 40 anos (22,2\%).

Realizou-se o acesso a um total de 1.815 exames VDRL do ano de 2017 , sendo que, dentre esses, $87,7 \%(1593 / 1815)$ mostraram resultado não reagente e $12,2 \%$ (222/1815) tiveram como resultado exames reagentes. Com relação aos exames realizados em 2018, acessou-se um total de 4.571 exames VDRL, sendo desses, 81,4\% (3721/4571) não reagentes e 18,5\% (850/4571) reagentes (Figura 2 ).

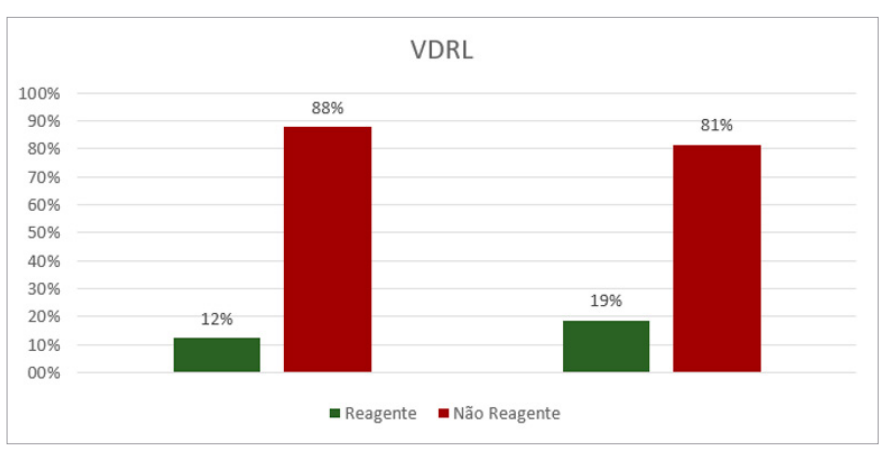

Figura 2: Percentual de pacientes reagentes e não reagentes ao teste não treponêmico VDRL em 2017 e 2018.

$\mathrm{Na}$ figura 3, estabeleceu-se o levantamento da relação entre os títulos dos pacientes reagentes ao exame VDRL em 2017 e em 2018, permitindo a observação das titulações mais frequentemente encontradas, através de curvas comparativas entre os anos estudados.

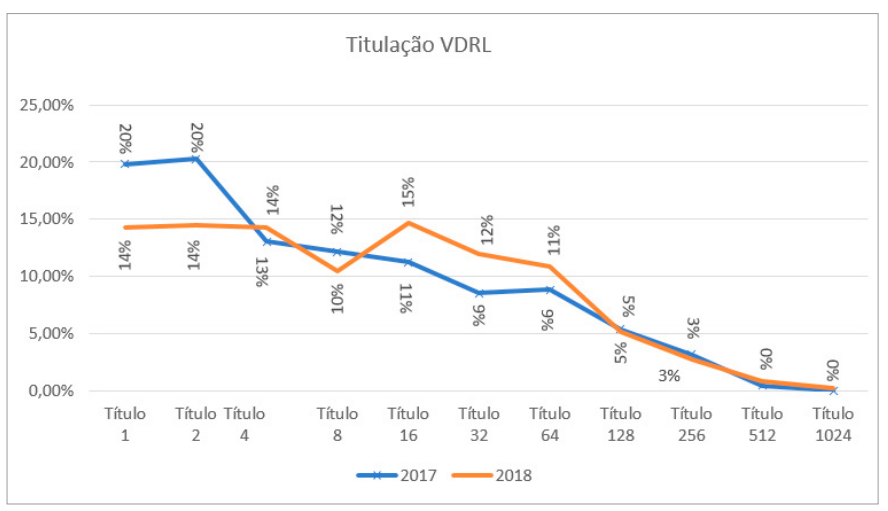

Figura 3: Curvas das prevalências de cada titulação encontrada nos exames VDRL Reagentes em 2017 e 2018. 
Dos pacientes submetidos à realização do exame VDRL em 2017 e que obtiveram resultado Reagente, 48,6\% (108/222) também realizaram a pesquisa do FTA-abs IgM e lgG. Quanto ao FTA-abs IgM, 46,3\% (50/108) tinham amostra reagente para a presença deste anticorpo, 34,2\% (37/108) tinham amostra não reagente para tal e 19,4\% (21/108) obtiveram o resultado indeterminado. Em relação à pesquisa de anticorpo FTA-abs lgG desta população, 91,7\% (99/108) tiveram resultado reagente e 8,3\% (09/108) obtiveram a resposta não reagente.

Com relação aos pacientes que realizaram o exame VDRL em 2018 e reagentes para tal, 16\% (136/850) também realizaram a pesquisa do FTA-abs IgM e 25,2\% (215/850) realizaram adjuntamente a pesquisa do FTA-abs IgG. Quanto ao FTA-abs $\operatorname{lgM}, 31,6 \%(43 / 136)$ tinham amostra reagente para a presença deste anticorpo, 8\% (11/136) tinham amostra não reagente para tal e 60,3\% (82/136) obtiveram como resultado a variável indeterminado para a presença do referido.

Considerando a pesquisa do anticorpo FTA-abs IgG desta população, 98,6\% (212/215) tiveram resultado reagente e 1,4\% $(03 / 215)$ obtiveram a resposta não reagente para a pesquisa deste anticorpo, conforme apresentado nas figuras 4 e 5 .

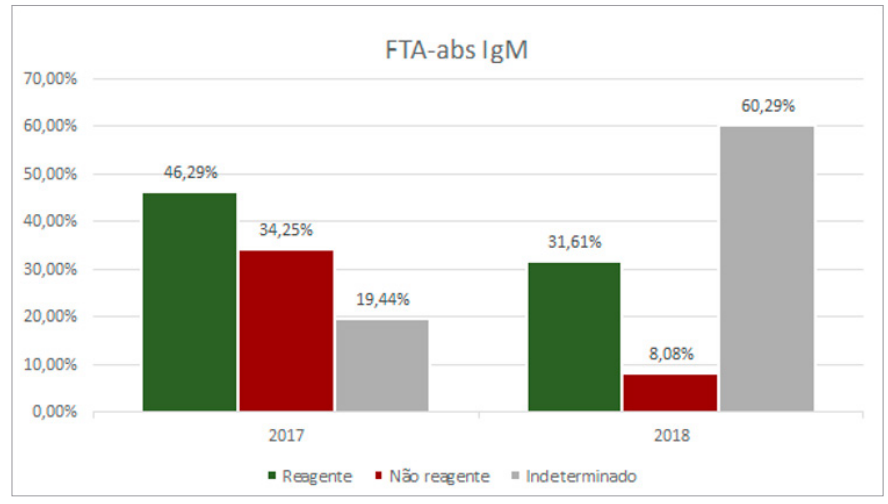

Figura 4: Resultados do exame FTA-abs IgM realizado em pacientes VDRL Reagentes em 2017 e 2018.

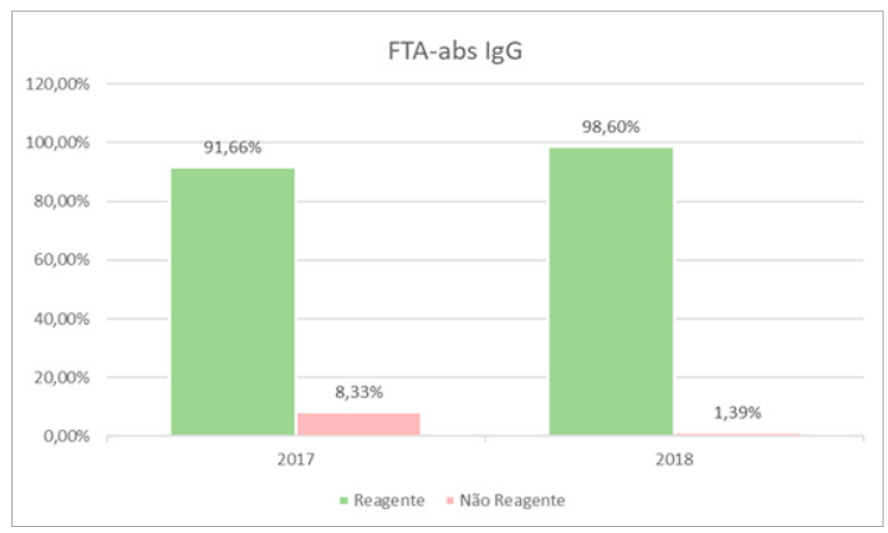

Figura 5: Resultados do exame FTA-abs IgG realizado em pacientes VDRL Reagentes em 2017 e 2018.

\section{Discussão}

A sífilis é um importante problema nacional de saúde pública, caracterizado pelo potencial infectocontagioso e por aumentar o risco de contrair a infecção de doenças sexualmente transmissíveis como o HIV. É uma doença de notificação compulsória regular, assim, todo caso confirmado deve ser notificado à Vigilância Epidemiológica conforme o critério: indivíduo assintomático com teste não treponêmico reagente com qualquer titulação e teste treponêmico reagente; ou clínica compatível, com pelo menos um teste reagente. Vale a ressalva de que a notificação e vigilância são imprescindíveis para interromper a cadeia de transmissão e indicar as medidas de controle necessárias.

Atualmente, o cenário epidemiológico da doença inclui o aumento da incidência, associado à subnotificação de $\operatorname{casos}^{10}$. Com relação a população alvo do presente estudo fora apresentado no total uma distribuição semelhante de exames analisados entre os sexos, com 3.355 pacientes do sexo masculino e 3.031 pacientes do sexo feminino. Entretanto, é notório o aumento no valor absoluto dos exames de triagem realizados ao se comparar a quantidade de pacientes referentes ao ano de 2017 com os de 2018, com um salto que vai de 1.815 exames realizados no primeiro para 4.571 realizados no segundo ano referido. Demonstrando assim, pela quase triplicação da solicitação de exames, um cenário clínico local com crescimento de demanda alarmante.

O estudo evidenciou uma elevação na triagem em pacientes do sexo masculino entre os anos de 2017 e 2018, indo de $36,6 \%$ dos exames realizados no primeiro para $58,8 \%$ no segundo ano referido. Já com relação à idade dos pacientes pesquisados ficou evidenciado o predomínio de adultos jovens, com média de idade de 37,9 anos em 2017 e 34,8 anos em 2018. A distribuição entre as idades de 31 a 40 anos se mostrou predominante em ambos os anos pesquisados, principalmente em 2018, chegando este intervalo de idade a compor $40,4 \%$ da totalidade dos pacientes investigados neste período.

Os testes não treponêmicos detectam anticorpos não específicos (anticardiolipina) para os antígenos do T. pallidum, são utilizados para rastreio de casos possíveis de sífilis, e podem ser qualitativos ou quantitativos ${ }^{11}$. Nesta pesquisa, levantouse os resultados do teste não treponêmico VDRL quantitativo, que permitiu evidenciar, ao se comparar os resultados dos exames referentes aos anos pesquisados, um crescimento de 6,36\% entre os exames com resultado reagente de 2017 e 2018. E, através da análise estatística, percebeu-se também uma diferença significativa nos resultados reagentes para o VDRL ao se comparar a população do sexo masculino com a do sexo feminino. Com diferença altamente significativa en- 
tre os sexos $(p<0,0001)$, observa-se na população masculina estudada um predomínio maior de reatividade ao VDRL e consequentemente uma maior possibilidade de infecção pelo Treponema pallidum.

Vale ressaltar que em 2014 houve diminuição da distribuição, em nível mundial, da penicilina benzatina, que é o principal medicamento usado para tratamento da síflis, fato este que configura um dos cernes principais do crescente número de casos que vem ocorrendo em todo o mundo?.

Com relação aos títulos encontrados nos exames VDRL reagentes, em 2017, 59,8\% dos pacientes se encontravam dentro dos valores estipulados como indicativos de infecção ( $\geq 1 / 4)$ e, dentre esses, encontrou-se com maior frequência a titulação correspondente a $1 / 4$ em um total de $14,2 \%$ dos casos. Por outro lado, em 2018, 71,3\% dos pacientes estavam com valores de títulos sugestivos de infecção, sendo o título 1/16 o mais prevalente na população, presente em $14,7 \%$ dos pacientes. Dessa forma, evidenciou-se um crescimento preocupante de $11,5 \%$ na possibilidade de infecção de acordo com o teste não treponêmico utilizado no rastreio, fato este associado à maior ocorrência de títulos mais altos no ano 2018.

Segundo recomendações do Ministério da Saúde, para o diagnóstico da síflis, devem ser utilizados, um dos testes não treponêmicos e um dos testes treponêmicos, sendo a ordem de realização desses acordada com cada serviço de saúde. Vale ressaltar que, em todo caso confirmado, deve ser realizada triagem para HIV e hepatites virais B e C devido à grande incidência de coinfecções por outras doenças sexualmente transmissíveis ${ }^{10}$. Entretanto, tornou-se evidente, pelos resultados do presente estudo, a quebra do ciclo de triagem diagnóstica de grande parte da população estudada.

Tal fato pôde ser demostrado pela baixa porcentagem dos pacientes que realizaram o teste não treponêmico (VDRL), e foram também submetidos ao teste treponêmico (FTA-abs). Visto que apenas $48,6 \%$ dos pacientes reagentes ao VDRL em 2017 foram submetidos à análise do FTA-abs IgM e lgG, e, em 2018, somente $16 \%$ dos pacientes reagentes aos VDRL foram submetidos à testagem do FTA-abs IgM, enquanto, no mesmo ano, apenas $25,2 \%$ dos pacientes foram triados também para a presença de FTA-abs IgG. Esta realidade elucida a inadequação do processo diagnóstico em mais da metade da população pesquisada em 2017 e em aproximadamente 75\% da população pesquisada em 2018.

Assim, sem a determinação dos anticorpos pesquisados pelo teste treponêmico, dificilmente houve o esclarecimento do curso clínico que o paciente apresentava na oferta de sua doença, levando a um alto nível de confusão entre as formas agudas e crônicas, que se associam a condutas terapêuticas semelhantes, mas divergentes em vários aspectos. Dessa forma, é notório o reflexo que tal cenário cria no tratamento, o qual, por esta realidade operante, certamente se desenvolverá da forma não esperada e sem resultados efetivos, tanto para a saúde do paciente em si, quanto para a tentativa de quebra do ciclo da doença e também para a prevenção do crescimento dos casos de sífilis no país ${ }^{10}$.

Com relação aos pacientes submetidos ao teste treponêmico FTA-abs IgM percebeu-se uma redução dos índices com resultado reagente entre os anos de 2017 e 2018 de $26,8 \%$ e redução dos exames com resultado não reagente de $26,2 \%$, associados ao aumento substancial de $40,9 \%$ dos exames com resultado indeterminado, fato este que possivelmente ocultou a realidade da população pesquisada. A baixa taxa de realização de rastreio do FTA-abs na população VDRL reagente também se apresenta como outra possibilidade para a redução não condizente apresentada. $E$, no que tange ao exame FTAabs IgG, encontrou-se um crescimento sutil esperado de $6,9 \%$ entre os anos de 2017 e 2018. Pelo fato de tal exame significar uma marca sorológica de contato crônico, esse crescimento se explica pelo aumento no número de casos desde 2014, um resultado então já esperado?.

\section{Conclusão}

É possível concluir, ao analisar as abordagens executadas durante a pesquisa, que a população encontra-se sujeita a um crescimento de infecção por síflis, principalmente os adultos jovens do sexo masculino. Tal fato advém das consequências de ações não congruentes às normas técnicas vigentes atuais, como a quebra do ciclo diagnóstico preconizado pelo Ministério da Saúde, a falta de distribuição mundial do medicamento utilizado no tratamento da afecção e a falta de conscientização dessa parcela da população.

Assim, tendo em mente o advento da internet e da acessibilidade digital e o consequente maior contato, do grupo populacional mais afetado pela infecção, com as noções de educação sexual e de medidas de prevenção das ISTs, fica estabelecido o grande desafio das políticas públicas atuais: garantir que um atual cenário repleto de informação e conhecimento se consagre em práticas efetivas para o controle da situação vigente.

Ademais, ressalta-se a necessidade de maiores estudos com delineamentos populacionais mais rígidos no intuito de especificar, de forma mais nítida e especifica, os subgrupos populacionais mais afetados pela doença, garantindo assim medidas de controle e prevenção direcionadas e efetivas. 


\section{Referências}

1. FMUSP. Clínica Médica. 2. ed. v. 7. 2016. p. 309.

2. Ros CT, Schmitt S. Global epidemiology of sexually transmitted diseases. 2008;110-114.

3. Galindo J, Mier JF, Alberto C, Carlos J. Neurosífilis: un problema antiguo que no pierde actualidad. Rev Colomb Psiquiatr. 2017; 1-8.

4. Lazarini FM, Barbosa DA. Educational intervention in Primary Care for the prevention of congenital syphilis. Rev. Latino-Am. Enfermagem. 2017; 2835-2845.

5. Secretaria de Vigilância em Saúde. Boletim Epidemiológico da Sífilis. Ministério da Saúde. 2017.

6. Acosta LMW, Gonçalves TR. Coinfecção HIV / sífilis na gestação e transmissão vertical do HIV : um estudo a partir de dados da vigilância epidemiológica. Pan Am J Public Heal. 2016;40:435-442.

7. Situação epidemiológica da síflis adquirida, sífilis em gestante e sífilis congênita no Estado de Goiás. Secr Estado da Saúde Goiás. 2017.

8. Trinh TT, Kamb ML, Luu M, Ham DC, Perez F. Syphilis testing practices in the americas. Tropical Medicine and International Healt. 2014;22(9):

9. Coelho JMR et al. Sífilis: um panorama epidemiológico do Brasil e do município de Volta Redonda/RJ. Brazilian J Heal Rev. 2018;1(1):128-147.

10. Pires Acs, Oliveira Dd, Rocha Gmnm, Santos A. Ocorrência de síflis congênita e os principais fatores relacionados aos índices de transmissão da doença no Brasil da atualidade - revisão de literatura. Rev Uningá Rev. 2014;19(1):58-64.

11. Reis GJ, Barcellos C, Pedroso MM, Xavier DR. Intraurban differentials in congenital syphilis: A predictive analysis by neighborhood in the city of Rio de Janeiro, Brazil. Cad Saude

Publica. 2018; 34-40. 